\title{
Spezifische Probleme beim Übersetzen von Phraseologismen am Beispiel des Kinderromans Emil und die Detektive von Erich Kästner
}

\author{
Jarosław Ślawski (Kraków)
}

\begin{abstract}
Specific problems of collocations translation illustrated on examples from children's novel Emil and the Detectives by Erich Kästner.

The paper presents specific problems of translating collocations based on examples from children's novel Emil and the Detectives and its four Polish translated versions.

These problems are due to the presence of collocations plentitude in everyday language and typical Kästner's modifications.

Kästner's modifications within collocations include metaphorical comparisons and the typical accumulation of collocations within a few verses.

Most often they are partly idiomatic and may cause difficulties for young readers with understanding. However, problematic matters are with zero equivalency counterparts in the target text, which are conditioned by external factors such as culture, history and folk customs. These factors should also be taken into consideration when it comes to a translation.
\end{abstract}

\section{$1 \quad$ Einführung}

Phraseologismen werden als sprachliche Einheiten bezeichnet, die sich unter Wortverbindungen durch Idiomatizität, Stabilität, Lexikalisierung und Reproduzierbarkeit auszeichnen (Fleischer 1997: 35ff). Auf Grund ihrer Merkmale können sie jedem Übersetzer Probleme bereiten.

Diese Probleme können damit verbunden sein, dass die phraseologische Semantik in der Kultur der jeweils untersuchten Sprache verwurzelt ist (vgl. Eismann 1995: 95-119).

Koller (1994: 351-357) behauptet, dass Phraseologismen zu solchen sprachlichen Einheiten gehören, die nur schwer oder überhaupt nicht in eine andere Sprache übertragbar sind.

Das Ziel der kontrastiven Untersuchung von phraseologischen Einheiten ist die Suche nach den äquivalenten Entsprechungen der Zielsprache für Phraseme der Ausgangssprache. Dementsprechend kann man verschiedene Strategien der Translation von Phraseologismen unterscheiden.

Werner Koller (1972: 171f.) nennt drei Möglichkeiten des Übersetzens von Phraseologismen: 
1. Wörtliche Übersetzung des $\mathrm{AS}^{1}$-Phraseologismus, die in der Zielsprache einen bedeutungsäquivalenten Phraseologismus ergibt,

2. "sinnentsprechende Interpretation" - Substitution durch einen fest zugeordneten Phraseologismus oder eine relativ sinnentsprechende Wendung,

3. nichtphraseologische Umschreibung des AS-Phraseologismus, wenn sich in der Zielsprache keine direkte phraseologische Entsprechung finden lässt:

a. lexikalische Paraphrase,

b. "Schein-Phraseologismen" (Neuschöpfungen), die aufgrund ihrer besonderen Form wie sprichwörtliche Redensarten klingen,

c. wörtliche Übersetzung.

Erika Worbs (1994: 165f.) sieht folgende Möglichkeiten:

1. Phraseologische Entsprechungen:

a. direkte phraseologische Äquivalente

b. analoge phraseologische Äquivalente

2. nichtphraseologische Entsprechungen:

a. Einwortlexeme

b. freie Sätze

c. stabile und freie Wortfügungen.

Für die Übersetzungsproblematik müssen vor allem Teiläquivalenz und Nulläquivalenz berücksichtigt werden. Diese zeigen die spezifischen Unterschiede zwischen zwei Sprachen.

Worbs unterscheidet auch (1994: 156f.) drei Hauptäquivalenzstufen:

1. Vollständige Äquivalenz, die eine Übereinstimmung zwischen AS- und ZS'2-Phrasem voraussetzt,

2. Partielle Äquivalenz, auch Teiläquivalenz, die bei einem vorauszusetzenden Mindestmaß an inhaltlicher Übereinstimmung auf der Nichtübereinstimmung in einem bzw. mehreren, meist miteinander zusammenhängenden verbundenen Faktoren beruht,

3. Nulläquivalenz, die darin besteht, dass in der Zielsprache keine systemhafte phraseologische Entsprechung für ein AS-Phrasem vorhanden ist, so dass sich Äquivalenz nur auf lexikalischer Ebene herstellen lässt.

In der vorliegenden Arbeit werden spezifische Probleme beim Übersetzen von Phraseologismen am Beispiel des Kinderromans Emil und die Detektive aufgrund seiner vier polnischen Übersetzungsversionen dargestellt. Als Kriterium der Analyse wurden folgende Elemente berücksichtigt: phraseologische Bedeutung, Idiomatizität, Art und Verwendung des Phraseologismus.

\footnotetext{
${ }^{1}$ AS - Ausgangssprache.

${ }^{2}$ ZS - Zielsprache.
} 
Jarosław Ślawski: Spezifische Probleme beim Übersetzen von Phraseologismen am Beispiel des 53 Kinderromans Emil und die Detektive von Erich Kästner

\section{Analyse der Phraseologismen im Kinderroman Emil und die Detektive}

Kästner verwendet in seinen Kinderbüchern "eine sehr interessante und originelle, milieuund lebensechte Sprache, die den Kindern abgelauscht wirkt, sehr natürlich und lebendig und von unnachahmlicher Prägnanz und Klarheit ist" (Sahr 1994: 34f.). Diese Merkmale, die in Kinderbüchern von Bedeutung sind, werden von Phraseologismen repräsentiert.

Dementsprechend zählt Burger (1997: 234) vier Typen des Gebrauchs von Phraseologismen auf: den abstinenten, den schonend-vermittelnden, den überbordend-hybriden, den spielerisch-augenzwinkernden und ordnet die Kindertexte von Kästner dem spielerischaugenzwinkernden Typ zu. Kinderbücher von Kästner sind für Burger (1997: 241) "Musterbeispiele von Phraseologiegebrauch auf mehreren Ebenen".

Schwerpunkte des Beitrags bilden: Verwendung umgangssprachlicher Phraseologismen, Phraseologische Vergleiche und Kästners phraseologische Variationen.

\subsection{Verwendung umgangssprachlicher Phraseologismen}

Kästner benutzt alltags- und umgangssprachliche Phraseologismen, um einen besseren Zugang $\mathrm{zu}$ jungen Lesern $\mathrm{zu}$ sichern. Die Verwendung umgangssprachlicher Phraseologismen, die in vergleichbarer Weise von Erwachsenen und Kindern, aber auch vom Erzähler selbst benutzt werden, dient der Personencharakteristik (Schatte 2003: 120).

Der Vergleich der phraseologischen Bedeutung am Beispiel der polnischen Übersetzungen von Emil und die Detektive kann Übersetzungsprobleme aufzeigen, die aufgrund von teiloder nulläquivalenten Phraseologismen entstehen. Trotzdem versuchen die polnischen Übersetzer größtenteils, einer phraseologischen Einheit der Ausgangssprache ein phraseologisches Äquivalent der Zielsprache zuzuordnen.

Beispiele für die Verwendung umgangssprachlicher Phraseologismen:

"Nun aber los, sagte Gustav, wenn wir nichts weiter machen als rumstehen, geht uns der Schuft durch die Lappen". [ED: 78] ${ }^{3}$

Das Dudenwörterbuch beinhaltet folgende Erklärungen für diese Redewendung: jemandem durch die Lappen gehen:"jemandem entkommen, entgehen". [Duden 2002: 461]

In den polnischen Wörterbüchern findet man dafür: jemandem durch die Lappen gehen $\rightarrow$ uciec <zwiać> komuś [GWDP: 12] und $\rightarrow$ wymknać się komuśs z rąk <sprzed nosa>. [SF: 255]

Die Übersetzung von Lazer gibt die Bedeutung des AS-Textes wieder.

"No, alle teraz jazda, do roboty - rzekł Gustaw - jeżeli nic nie zrobimy dalej, tylko będziemy tutaj ślęczeli, łotr gotów nam się wymignąć". [EDL: 119]

Lazer ersetzt den Phraseologismus der AS durch einen bedeutungsäquivalenten in der ZS gotów nam się wymignać, was den expressiven Charakter des Phraseologismus bestätigt, wymignać <wykręcić się od czegoś $>$ [SFP: 667] $\rightarrow$ drücken sich vor.

\footnotetext{
${ }^{3}$ Erklärung aller Siglen im Quellenverzeichnis. In allen Zitaten im Text ist die originale Schreibung beibehalten.
} 
Stroynowski übersetzt diese Phrase folgendermaßen:

"No więc jazda - powiedział Gustaw.- Jeżeli będziemy tu stali bezczynnie, ten łobuz nam się wymknie". [EDS:103]

Stroynowskis Übersetzung stimmt mit dem Original überein, (wymknąć się $\rightarrow$ entschlüpfen).

Auch Stroynowski benutzt in seiner Übersetzung einen bedeutungsäquivalenten Phraseologismus, um die phraseologische Phrase der AS wiederzugeben.

In Gradsteins Übersetzung finden wir eine originelle Paraphrase:

"A teraz ruszajmy - powiedział Gustaw. - Jeżeli dalej będziemy tu stać bezczynnie, to ten łajdak się nam wykruszy". [EDG:91]

Das Verb wykruszyć $\rightarrow$ abbröckeln hat in Bezug auf Menschen eine bildhafte Bedeutung, die mit der phraseologischen Bedeutung des AS-Textes übereinstimmt.

Wenn innerhalb einer kurzen Textpassage zwei oder mehr Phraseologismen auftreten, können wir von Phraseologismenhäufung sprechen.

Der phraseologische Charakter der Wortverbindungen ist in Bezug auf den Text ein stilistisches Mittel, das der Betonung oder Hervorhebung einer Textpassage dient, und das in der Figurenrede zu einem Sprachporträt beitragen kann (Richter-Vapaatalo 2007: 103).

Folgendes Beispiel beinhaltet eine Phraseologismenhäufung: Die Redewendung nicht von der Pelle gehen wurde der Volkssprache entnommen. Aus der Umgangssprache stammt die zweite Redewendung was ausgefressen haben:

"Ach, Mensch, da hat einer was ausgefressen und dem gehen wir nicht mehr von der Pelle - erklärte Gustav". [ED: 94]

In den deutschen Wörterbüchern gibt es folgende Erklärungen für diese Redewendungen:

1) etwas ausgefressen haben (ums.):"etwas angestellt, verbrochen haben". [Duden 2002: 78] und etwas ausgefressen haben:"heimlich eine Straftat, etwas schlechtes begangen haben". [LSR: 119]

2) jemandem nicht von der Pelle gehen (salopp): "jemanden nicht in Ruhe lassen, mit seiner dauernden Anwesenheit lästig fallen". [Duden 2002: 96]

In den polnischen Wörterbüchern gibt es folgende Übersetzungsvorschläge: jemandem nicht von der Pelle gehen $\rightarrow$ siedzieć komuś na karku [SF: 339], und $\rightarrow$ nie odstępować kogoś na krok. [GWDP: 219]

Lazers Übersetzung stimmt mit der Bedeutung des AS-Textes überein:

"Ach bracie, jeden tu coś przeskrobal, i nie ustąpimy mu już z fleku - wyjaśnił Gustaw". [EDL: 145]

In Lazers Übersetzung wurde die Redewendung was ausgefressen haben durch sein bedeutungsäquivalentes Verb in der ZS przeskrobać ersetzt, was den expressiven Charakter des Phraseologismus bestätigt. Die zweite Redewendung wurde mit Hilfe einer Paraphrase nie ustapimy mu już z fleku 'wir rühren uns nicht vom Flecke' übersetzt. 
Jarosław Ślawski: Spezifische Probleme beim Übersetzen von Phraseologismen am Beispiel des 55 Kinderromans Emil und die Detektive von Erich Kästner

Stroynowski übersetzt folgendermaßen:

"Ach, proszę pana, tamten facet coś przeskrobal, a my depczemy mu po piętach - wyjaśnił Gustaw". [EDS:122]

Stroynowski ersetzte die zweite Redewendung des AS-Textes durch einen Phraseologismus my depczemy mu po piętach <chodzić za kimś natrętnie, żeby go śledzić> [SFP: 168] $\rightarrow$ wir sind ihm auf den Fersen <jemandem auf Schritt und Tritt folgen um ihm nachzuspüren>, die mit der Bedeutung des Originals übereinstimmt.

Eine ähnliche Übersetzung schuf Gradstein.

Eine originale Übersetzung findet man in Kuszyks Übersetzung:

"Pszepana, przed nami jedzie taki jeden, co strasznie nabroil. Musimy go mieć na oku wyjaśnił Gustaw". [EDK:85]

Die Übersetzung von Kuszyk gibt die Bedeutung des AS-Textes wieder, obwohl sie einige Modifikationen enthält. Es handelt sich um eine Expansion durch das Adverb strasznie' furchtbar', das den phraseologischen Charakter verstärkt. Der Phraseologismus was ausgefressen haben wurde durch ein bedeutungsäquivalentes in der ZS Verb coś nabroić $\rightarrow$ etwas angestellt haben ersetzt. Kuszyk gibt die zweite Redewendung des AS-Textes durch einen Phraseologismus wieder: musimy go mieć na oku [SFP:588] 'wir müssen ihn im Auge behalten', der mit der Bedeutung des Originals übereinstimmt.

Neben einer Überfülle von Verben aus der Umgangssprache finden sich in allen Werken Kästners solche aus den verschiedensten Mundarten, Berufs- und Standessprachen z.B.: hoppnehmen aus der Gaunersprache (Bossmann 1955: 81).

Ein Gespräch zwischen Emil und Gustav ist ein gutes Beispiel für die Gaunersprache:

"Sag's doch dem Schuppo dort. Der nimmt ihn hopp". [ED: 78]

Das Dudenwörterbuch gibt folgende Erklärungen für diese Redewendung: hoppnehmen (salopp): "verhaften, festnehmen, ausbeuten".[DUW: 848]

Die Substitution von festnehmen durch hoppnehmen passt das Bild einerseits an den Kontext an, andererseits bildet es einen komischen, zweideutigen Effekt durch die Verwendung des Verbs aus der Gaunersprache.

Ein bedeutungsäquivalentes Verb in der Zielsprache verwendet Stroynowski:

"Powiedz policjantowi, który tam stoi. Ten go przyskrzyni". [EDS:102]

Das Verb przyskrzynić 'einsperren' bestätigt den expressiven Charakter des ZS-Textes.

Kuszyk übersetzt diese Phrase folgendermaßen:

"Powiedz żandarmowi. O, tam stoi. Już on się nim zajmie". [EDK:73]

Die von Kuszyk vorgeschlagene Übersetzung durch die Paraphrase klingt eher positiv, was mit der Bedeutung des AS-Textes nicht übereinstimmt zajać się 'sich beschäftigen mit'.

Eine abweichende Übersetzung finden wir bei Gradstein. Sie fand einen bedeutungsäquivalenten Phraseologismus in der Zielsprache zatatwić kogoś <obstugiwać kogoś w jakiejśs sprawie $>$ [SFP: 749] 'erledigen, fertig machen'<jemanden bedienen>. 
"Zawołaj policjanta, stoi tam na rogu. Ten go zaraz zalatwi". [EDG: 90]

Und Lazer übersetzt so:

"Powiedz przecież o tem temu tam policjantowi. On go już weźmie do galopu". [EDL:118]

Diese Paraphrase bedeutet, dass der Polizist (auf Gustavs Verlangen) den Dieb verfolgen soll, (do galopu wziać $\rightarrow$ verfolgen). Im AS-Text handelt es sich um die Festnahme eines Diebes.

\subsection{Phraseologische Vergleiche}

Phraseologische Vergleiche sind "bildhafte Ausdrücke", die sich in der Sprache als phraseologische Einheiten herausgebildet haben und in den lexikalischen Bestand einer gegebenen Sprache eingegangen sind (Militz 1982, zit. nach Szczęk 2010: 72).

Phraseologische Vergleiche hat Kästner benutzt, um die Eigenschaften und Verhaltensweisen von Personen $\mathrm{zu}$ beschreiben. Meistens sind sie teilidiomatisch und können den jungen Lesern Verständnisprobleme bereiten. Darüber hinaus werden sie anschaulich, humorvoll, witzig und in Alltagssituationen eingebettet, was für die jungen Leser attraktiv ist.

Beispiele für phraseologische Vergleiche:

"Ducken! Flüsterte Gustav. Die Jungen warfen sich zu Boden und lagen wie Kraut und Rüben durcheinander". [ED: 95]

In Lazers Übersetzung haben wir eine Wiedergabe des AS-Idioms durch ein auf der Ebene des allgemeinen Sinns äquivalentes ZS-Idioms, wo das Substantiv die Rüben 'buraki' durch groch 'Erbsen' ersetzt wurde.

"Schować się! szepnął Gustaw. Chłopcy rzucili się plackiem na dno samochodu, i leżeli po chwili jak groch z kapustą, jeden poprzez drugiego". [EDL: 145]

Lazers Übersetzung stimmt fast mit der Übersetzungserklärung von Donath überein [WI: 106]: Es geht alles wie Kraut und Rüben durcheinander $\rightarrow$ być pomieszane <leżeć> jak groch z kapusta.

Auch Stroynowskis Übersetzung gibt die Bedeutung des AS-Textes wieder, obwohl sie sich bildlich unterscheidet - "Kryć się - szepnął Gustaw. Chłopcy rzucili się plackiem na dno samochodu i leżeli tam stloczeni jak śledzie w beczce" [EDS: 124] 'lagen dort wie die Heringe zusammengepresst'.

Stroynowskis Übersetzung stimmt fast mit der Erklärung des Phraseologismus von Skorupka überein [SFP: 301]: "tłoczyć się jak śledzie w beczce".

Gradstein bevorzugt in ihrer Übersetzung eine einfache Wiedergabe des AS-Textes:

"Chować się - szepnął Gustaw. Chłopcy padli na podłogę i leżeli jeden na drugim jak worki" [EDG: 108] 'lagen wie Säcke aufeinander'.

Kuszyk verwendet in ihrer Übersetzung eine Reduktion, die als eine kontextbedingte Weglassung des AS-Phraseologismus bezeichnet wird. Sie verzichtete ganz auf die Form lagen wie Kraut und Rüben durcheinander:

"Kryć się! - wyszeptał nagle Gustaw. Chłopcy rzucili się na podłogę samochodu". [EDK: 86] 
Jarosław Ślawski: Spezifische Probleme beim Übersetzen von Phraseologismen am Beispiel des 57 Kinderromans Emil und die Detektive von Erich Kästner

Zur Beschreibung des Verhaltens seiner Romanfiguren benutzt Kästner metaphorische Phraseologismen. Sie werden als stilistisches Mittel in bestimmten Textsituationen verwendet. Metaphern sind sprachliche Bilder, die auf einer Ähnlichkeitsbezeichnung zwischen zwei Gegenständen basieren. Mit Hilfe von ähnlichen oder gleichen Bedeutungsmerkmalen entdeckt man eine Bezeichnungsübertragung der Metapher (Bußmann 2002: 432).

Beispiele für metaphorische Phraseologismen:

"Mir stehen die Haare zu Berge, obwohl ja alles längst vorüber ist". [ED: 157]

Die Bedeutung der Phrase lässt sich aus den Bedeutungen der einzelnen Bestandteile nicht ableiten. Aus diesem Grunde wurde von polnischen Übersetzern in jedem Fall der Versuch unternommen, einer phraseologischen Einheit der Ausgangssprache ein phraseologisches Äquivalent der Zielsprache zuzuordnen.

Diese Phrase stimmt mit der Dudenerklärung überein: jemandem stehen die Haare zu Berge $\rightarrow " j e m a n d e m$ sträuben sich die Haare, jemandem ist in höchstem Maß erschrocken, entsetzt". [Duden 2002: 305]

Im Falle von Stroynowskis Version haben wir eine Übersetzung des AS-Phraseologismus, die in der Zielsprache einen bedeutungsäquivalenten Phraseologismus bildet.

"Wlosy dęba mi stoją, gdy o tym pomyślę, chociaż już po wszystkim". [EDS: 175]

Gradsteins Übersetzung unterscheidet sich in der Wortfolge bei Stroynowski: "Włosy stoją mi dęba, chociaż to wszystko już minęło". [EDG: 175]

Lazer hat den ZS-Text mit Hilfe um ein Adverb po prostu 'einfach' bereichert, wobei die phraseologische Semantik keinen Schaden davon trägt.

"Po prostu włosy mi na głowie stoją, choć przecież dawno już po wszystkiem". [EDL: 239]

Kuszyk verwendet einen bedeutungsäquivalenten Phraseologismus: "Włosy jeżą mi się na głowie na samą myśl, chociaż już jest po wszystkim" [EDK: 132]. Die Redewendung włosy jeżą mi się na głowie entspricht der Dudenerklärung $\rightarrow$ die Haare sträuben sich.

\subsection{Kästners phraseologische Variationen}

Kästner nutzt in seinem spielerischen Umgang mit Phraseologismen alle üblichen Verfahren des Sprachspiels, angefangen mit der einfachen Einbettung in den Kontext bis zu diversen Modifikationen der Form und Komponentenkette (Schatte 2003: 124).

Kästners Modifikationen der Phraseologismen erläutern nicht nur den Umgang mit festen Verbindungen sondern stellen auch ihre strukturellen Veränderungen mit ihrer Bedeutung dar. Die Verbreitung der phraseologischen Komponenten kann vor allem humorvolle Effekte erzeugen, auch wenn sich zusätzliche Lexeme, die eingefügt wurden, nicht in das phraseologische Bild passen. Folgende Beispiele zeugen von diesen Besonderheiten:

"Hütchen nahm wieder ihr Rad und inspizierte den Bahnhof. Es gab weiter keinen zweiten Blumenstand. Dann fragte sie noch rasch zwei Eisenbahnbeamten Löcher in den Bauch und kam stolz zurück". [ED: 72] 
Diese Phrase hat folgende Dudenerläuterung: jemandem ein Loch/Löcher in den Bauch fragen, salopp:"jemandem pausenlos Fragen stellen".[Duden 2002: 519]

Im Falle Gradsteins Version haben wir eine wortwörtliche und bildhafte Übersetzung des ASPhraseologismus, die in der Zielsprache einen bedeutungsäquivalenten Phraseologismus ergibt: "Pony Kapelusik wzięła znów rower i obeszła dworzec. Nie było drugiego kiosku z kwiatami. A potem wywiercila jeszcze pytaniami dziurę w brzuchu dwóm innym urzędnikom kolejowym i dumna wróciła do babci". [EDG: 86]

Gradsteins Übersetzung stimmt fast mit der Übersetzungserklärung von Czochralski überein [SW: 273]: jemandem ein Loch/Löcher in den Bauch reden <fragen> ugs. $\rightarrow$ wiercić komuś dziurę w brzuchu o coś.

Das ist auch ein Beispiel für die grammatische Modifikation in dem ZS-Text, die Gradstein bevorzugt. Das Verb fragen wurde im ZS-Text durch das Substantiv im Plural pytaniami 'mit Fragen' ersetzt. Das Substantiv im Plural Löcher wurde im ZS-Text durch das perfektive Verb wywierciła 'hat ausgebohrt' ersetzt.

Im Falle der übrigen polnischen Versionen haben wir eine Wiedergabe des AS-Idioms durch eine Paraphrase. Kuszyk übersetzt sie folgendermaßen:

"Kapelusik chwyciła rower i udała się na obchód dworca. Ale drugiej kwiaciarni nie znalazła. Potem pomęczyła jeszcze pytaniami dwóch pracowników kolei i wreszcie, puchnąc z dumy, wróciła do babci" [EDK: 68]. Kuszyk fand eine einfache Wiedergabe für die Bedeutung des AS-Textes: pomęczyć jeszcze pytaniami 'jemanden mit Fragen quälen'.

Denselben Weg wählen auch Stroynowki und Lazer:

"Pony Kapelusik zabrała swój rower i spenetrowała dworzec. Drugiego kiosku z kwiatami nie było. Potem jeszcze szybko zanudzila na śmierć pytaniami dwóch kolejarzy i wreszcie bardzo z siebie dumna powróciła do babci" [EDS: 96]: zanudzić na śmierć pytaniami $\rightarrow z u$ Tode mit Fragen langweilen.

Kästner benutzt auch Substitutionen, deren komische Wirkung nur bei Kenntnis der Ausgangsstruktur des Phraseologismus verstanden wird.

"'Na ja', erzählte Pony, 'die Eltern und Großmutter sitzen nun zu Haus und bohren sich Löcher in den Kopf, was mit dir eigentlich los ist"'. [ED: 101]

In dieser phraseologischen Modifikation spielt das Verb bohren eine große Rolle. In seinem idiomatischen Bereich bedeutet bohren:"etwas nachgrübeln, etwas zu ergründen, suchen". [WdU: 121]

Einen gelungenen Versuch der Übersetzung für die Wiedergabe des AS-Ausdrucks durch eine Paraphrase macht Lazer:

"No tak, - odpowiadała Polcia Kapelusik - rodzice moi i babcia siedzą w domu i dziurawią sobie mózg, co się to właściwie z tobą stało". [EDL: 153]

Die Paraphrase Lazers stimmt mit der Bedeutung des AS-Textes überein. In Lazers Übersetzung wurde das Verb bohren durch ein äquivalentes Verb in der ZS dziurawić ersetzt. Das Substantiv der AS der Kopf wurde durch das Substantiv mózg 'das Gehirn' in der ZS ersetzt. Das Substantiv Löcher wurde im ZS-Text weggelassen. 
Jarosław Ślawski: Spezifische Probleme beim Übersetzen von Phraseologismen am Beispiel des 59 Kinderromans Emil und die Detektive von Erich Kästner

Stroynowski benutzt in seiner gelungenen Übersetzung einen bedeutungsäquivalenten Phraseologismus (łamać sobie głowy), die eine Sing-P1-Modifikation des ZS-Textes ist:

"No, tak - ciągnęła Pony - rodzice i babcia siedzą teraz w domu i lamią sobie głowy, co się z tobą właściwie dzieje". [EDS: 128]

Das Substantiv der AS der Kopf wurde durch das Substantiv głowy (P1.) 'Köpfe' in der ZS ersetzt. Darüber hinaus wurde das Verb der AS bohren durch das Verb der ZS łamać 'brechen' ersetzt.

Eine zusätzliche Erklärung des Phraseologismus tamać sobie głowy finden wir bei Skorupka <mysleć o czymś bardzo intensywnie, kłopotać się czymś> [SFP: 243] 'an etwas sehr intensiv denken, sich sorgen um etwas'.

In Gradsteins Übersetzung haben wir ein Beispiel für die Wiedergabe des AS-Ausdrucks durch ein einfaches ZS-Lexem, was zur Reduktion führt.

"No tak - mówiła Pony dalej. Rodzice i babcia siedzą teraz w domu i nie mogą pojąć, co się z tobą dzieje". [EDG: 112]

Das Verb der AS bohren wurde durch das Verb der ZS pojać 'begreifen' ersetzt.

In Kuszyks Übersetzung haben wir ein Beispiel der Wiedergabe des AS-Ausdrucks durch ein polnisches Idiom:

"Ale kino - mówiła dalej Pony Kapelusik. Rodzice z babcią siedzą w domu i zachodzą w głowę, co takiego ci się właściwie stało". [EDK: 90]

Eine zusätzliche Erklärung des idiomatischen Ausdrucks zachodzić w głowę finden wir bei Skorupka <zastanawiać się, dziwić się, zdumiewać się, nie rozumieć> [SFP: 721] 'überlegen, wundern, erstaunen, nich verstehen'.

Einige Redewendungen aus der Umgangssprache hat Kästner abgeändert, meistens durch die Einführung eines anderen Verbums: hart verpackt sein $\rightarrow$ für zugeknöpft sein (Bossmann 1955: 80). Diese Modifikation wird noch verstärkt und auffällig durch die Erweiterung um ein Adverb:

"'Alle Wetter, Heimbold, bist du ein Dickschädel', sagte Pony Hütchen zu ihrem Vater. Siehst du denn nicht, daß Emil sich so darauf freut, seiner Mutter was zu schenken? Ihr Erwachsenen seid manchmal kolossal hart verpackt"'.[ED: 164]

In den polnischen Versionen wird der AS-Phraseologismus äquivalent wiedergegeben:

Kuszyk übersetzt folgendermaßen: "Wy dorośli jesteście czasami strasznie gruboskórni".[EDK: 137]

Die gelungene Übersetzung Kuszyks stimmt mit der Bedeutung der AS überein: gruboskórny $\rightarrow$ dickfellig. Das Adverb strasznie 'furchtbar' verstärkt den Effekt.

Skorupka erklärt den idiomatischen Ausdruck być gruboskórnym folgendermaßen: <być niedelikatnym, pozbawiony taktu, ordynarnym > [SFP: 265] 'grob, taktlos, ordinär sein'.

Im Falle von Stroynowskis Übersetzung haben wir eine leichte Abschwächung der phraseologischen Bedeutung. Dafür ist das Adjektiv niedomyślny 'begriffsstuzig' verantwortlich: "Wy, dorośli, bywacie czasami strasznie niedomyślni". [EDS: 210] 
Gradstein zeigt in ihrer Übersetzung mehr Originalität, trotzdem beinhaltet sie die Bedeutung des Ausgangstextes: "Wy dorośli to czasem macie zakute głowy". [EDG:180]

Gradstein bevorzugt in ihrer Übersetzung eine Wiedergabe des AS-Idioms durch ein bedeutungsäquivalentses ZS-Idiom mit der grammatischen Modifikation, in der ein Verb mieć 'haben' eingesetzt wurde, und die Wendung hart verpackt durch zakute głowy 'geschmiedete Köpfe' als Ausdruck metaphorischen Phraseologismus ersetzt wurde.

\section{$3 \quad$ Zusammenfassung}

Der deutsch-polnische Vergleich der phraseologischen Bedeutung am Beispiel des Kinderromans Emil und die Detektive zeigt Übersetzungsprobleme auf, die aufgrund von teiloder nulläquivalenten Phraseologismen entstehen.

Viele Probleme ergeben sich vor allem aus einer Überfülle umgangssprachlicher Phraseologismen, aus phraseologischen Vergleichen und aus phraseologischen Variationen.

Die polnischen Übersetzer haben folgendes Verfahren angewandt, um diese Übersetzungsprobleme zu lösen.

1. Bei der Verwendung umgangssprachlicher Phraseologismen versuchten sie einer phraseologischen Einheit der Ausgangssprache einen bedeutungsäquivalenten Phraseologismus in der Zielsprache zuzuordnen. Im Falle der Phraseologismenhäufung gelang es Lazer die Stilelemente und den expressiven Charakter der Redewendung des Textes zu erhalten. Die übrigen polnischen Übersetzungsvorschläge versuchten das AS-Idiom durch ein polnisches, äquivalentes ZS-Idiom wiederzugeben. Dieses Verfahren kann zum Wechsel der Stilschicht führen, was Kuszyks Übersetzung beweist.

Übersetzungsprobleme bereiten vor allem Phraseologismen, die auf verschiedensten Mundarten, Berufs- und Standessprachen basieren. Ein gutes Beispiel für die gelungene Übersetzung der Redewendung der Gaunersprache Der nimmt ihn hopp wird in Stroynowskis Übersetzung dargestellt, wobei die übrigen polnischen Übersetzungsversionen in diesem Fall weniger gelungen sind.

2. Das zweite Übersetzungsproblem bereiten phraseologische Vergleiche, die in der ZS als sinnentsprechende, äquivalente Idiome übersetzt werden (Lazer) was ermöglicht, anschauliche, humorvolle und in Alltagsituationen eingebettete Ausdrucksweise widerzuspiegeln. Bei übrigen polnischen Übersetzungsvarianten wird die Bedeutung des ASTextes durch das unterschiedliche, ausgebaute, sprachliche "Bild" erhalten. Im Gegensatz dazu verwendet Kuszyk in ihrer Übersetzungsvariante eine Reduktion des ganzen AS-Idioms, was zum Wechsel der Stilschicht führt.

3. Das letzte Übersetzungsproblem bereiten Kästners phraseologische Variationen. Polnische Übersetzer haben folgendes Verfahren angewandt: eine wortwörtliche und bildhafte Übersetzung des AS-Phraseologismus, grammatische Modifikationen und eine Wiedergabe des AS-Idioms durch eine Paraphrase.

Kästner bevorzugt in seinem Umgang mit Phraseologismen alle mögliche Verfahren des Sprachspiels, angefangen mit umgangssprachlichen Phraseologismen, dann mit phraseologischen Vergleichen bis zu phraseologischen Variationen. Um Kästners Sprachspiel 
Jarosław Ślawski: Spezifische Probleme beim Übersetzen von Phraseologismen am Beispiel des 61 Kinderromans Emil und die Detektive von Erich Kästner

wiederzugeben, müssen Phraseologismen im Übersetzungsprozess komplex mit ihren außersprachlichen und innersprachlichen Besonderheiten betrachtet werden.

\section{Korpusverzeichnis (mit Siglen)}

Czochralski, Jan, Klaus-Dieter Ludwig (1999): Słownik frazeologiczny niemiecko-polski. Warszawa. [SF]

Donath, Adolf (1975): Wybór idiomów. Warszawa [WI]

Duden (2002): Duden. Redewendungen. Wörterbuch der deutschen Idiomatik. 2., neu bearbeitete und aktualisierte Auflage, Mannheim: Dudenverlag. [Duden]

Duden (2007): Deutsches Universalwörterbuch. 6., Auflage, Mannheim: Dudenverlag. [DUW]

Kästner, Erich (1990): Emil und die Detektive. 135., Auflage, Hamburg: Cecilie Dressler Verlag. [ED]

Kästner, Erich/ Gradstein, Leonia (1980): Emil i detektywi. 4 Auflage, Warszawa: Świat Książki. [EDG]

Kästner, Erich/ Kuszyk, Karolina (2010): Emil i detektywi. 3., Auflage, Toruń: C\&T. [EDK]

Kästner, Erich/ Lazer, Dawid (1933): Emil i detektywi. 2., Auflage, Kraków: Księgarnia Powszechna. [EDL]

Kästner, Erich/ Stroynowski, Juliusz (1957): Emil i detektywi. 1., Auflage, Warszawa: Nasza Ksiegarnia. [EDS]

Küpper Heinz (1992): Wörterbuch der deutschen Umgangssprache. Leipzig: VERLAG. [WDU]

Piprek, Jan/ Ippoldt, Juliusz (2001): Großwörterbuch Deutsch-Polnisch. 2 Bde. Warszawa: Wiedza Powszechna. [GWDP]

Röhrich, Lutz (1991): Das große Lexikon der sprichwörtlichen Redensarten. Band 1, Freiburg: Herder. [LSR]

Skorupka, Stanisław (1977): Słownik frazeologiczny języka polskiego. Warszawa: Wiedza Powszechna. [SFP]

\section{Literatur}

Bossmann, Reinaldo (1955): Erich Kästner. Werk und Sprache. Curitiba: Haupt.

Burger, Harald (1997): "Phraseologie im Kinder- und Jugendbuch". In: Wimmer, Rainer/Berens, Franz-Josef (eds.): Wortbildung und Phraseologie. Tübingen, Narr: 234241.

Bußmann, Hadumod (2002): Lexikon der Sprachwissenschaft. 3., aktualisierte Auflage, Stuttgart: Kröner.

Eismann, Wolfgang (1989): "Zum Problem der Äquivalenz von Phraseologismen". In: Gréciano-Grabner, Gertrud (ed.): Europhras 88. Phraseologie contrastive. Strasbourg, Université des scienceshumaines. Département d' étudesallemades: 83-93.

Fleischer, Wolfgang (1997): Phraseologie der deutschen Gegenwartssprache. 2. Auflage, Tübingen: Niemeyer.

Koller, Werner (1972): Grundprobleme der Übersetzungstheorie. Bern etc.: Francke.

Koller, Werner (1994): "Phraseologismen als Übersetzungsproblem". In: Sandig, Barbara(ed.): Europhras 92. Tendenzen der Phraseologieforschung. Bochum, Universitätsverlag Dr. N. Brockmeyer: 351-373. 
Richter-Vapaatalo, Ulrike (2007): Das hatte das Pferd die Nüstern voll. Gebrauch und Funktion von Phraseologie im Kinderbuch. Untersuchungen zu Erich Kästner und anderen Autoren. Frankfurt a. M.: Lang.

Sahr, Michael (1994): 5x Kinderbücher im Unterricht. Möglichkeiten im Umgang mit Kinderliteratur in der Grundschule. Baltmannsweiler: Schneider.

Schatte, Czesława (2003): "Zur Verwendung der Phraseologismen in Kinder- und Jugendromanen von Erich Kästner". In: Mielczarek, ZygmuntWeigt, Zenon (eds.): Literatur und Linguistik. Germanistische Studien. 2. Band, Częstochowa, 118-128.

Szczęk, Joanna (2010): Auf der Suche nach der phraseologischen Motiviertheit im Deutschen (am lexikographischen Material). Dresden etc.: Neisse.

Worbs, Erika (1994): Theorie und Praxis der slawisch-deutschen Phraseographie. Mainz: Liber. 